\title{
Sub-10 fs Time-Resolved Vibronic Optical Microscopy
}

Christoph Schnedermann, ${ }^{\dagger}$ Jong Min Lim, ${ }^{\dagger}$ Torsten Wende, ${ }^{\dagger}$ Alex S. Duarte, ${ }^{\dagger, \S}$ Limeng Ni, ${ }^{\ddagger}$ Qifei Gu, Aditya Sadhanala, ${ }^{\ddagger}$ Akshay Rao, ${ }^{\ddagger}$ and Philipp Kukura* ${ }^{\dagger}$

${ }^{\dagger}$ Physical and Theoretical Chemistry Laboratory, University of Oxford, South Parks Road, Oxford OX1 3QZ, United Kingdom

${ }^{\ddagger}$ Cavendish Laboratory, University of Cambridge, JJ Thomson Avenue, Cambridge CB3 0HE, United Kingdom

\section{Supporting Information}

ABSTRACT: We introduce femtosecond wide-field transient absorption microscopy combining sub-10 fs pump and probe pulses covering the complete visible (500-650 $\mathrm{nm})$ and near-infrared (650-950 nm) spectrum with diffraction-limited optical resolution. We demonstrate the capabilities of our system by reporting the spatiallyand spectrally-resolved transient electronic response of $\mathrm{MAPbI}_{3-x} \mathrm{Cl}_{x}$ perovskite films and reveal significant quenching of the transient bleach signal at grain boundaries. The unprecedented temporal resolution enables us to directly observe the formation of bandgap renormalization, completed in 25 fs after photoexcitation. In addition, we acquire hyperspectral Raman maps of TIPS pentacene films with sub-400 $\mathrm{nm}$ spatial and sub- 15 $\mathrm{cm}^{-1}$ spectral resolution covering the $100-2000 \mathrm{~cm}^{-1}$ window. Our approach opens up the possibility of studying ultrafast dynamics on nanometer length and femtosecond time scales in a variety of two-dimensional and nanoscopic systems.
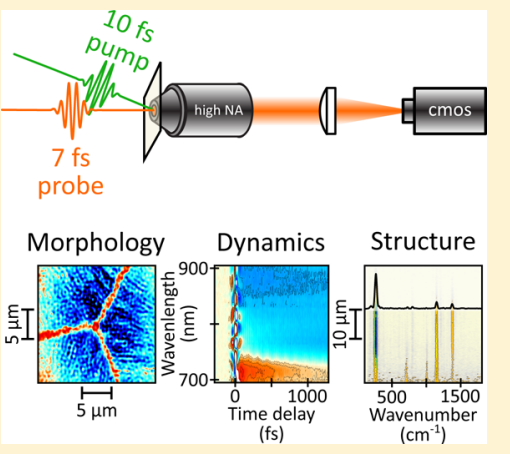

$\mathrm{N}$ anostructured electronic materials such as organic semiconductors $^{1-3}$ and organic-inorganic metal halide perovskites ${ }^{4-6}$ have attracted considerable attention both from the point of view of fundamental electronic properties and for applications in next-generation photovoltaics (PVs), lightemitting diodes (LEDs), and other optoelectronic devices. In contrast to conventional semiconductors such as silicon, one of the defining features of these materials is the spatial inhomogeneity of thin films on $\mu \mathrm{m}$ and sub- $\mu \mathrm{m}$ length scales. This has been widely studied by electron, X-ray and photoluminescence (PL) microscopy, ${ }^{7-14}$ yet an understanding of how these structural and chemical properties control and influence exciton and charge dynamics remains unknown. This lack of understanding can be largely traced back to the fact that transient electronic and vibrational spectroscopies are traditionally and predominantly performed at the ensemble level due to the necessity of combining the response from a significant amount of material to generate an observable signal in the presence of measurement noise. Critically, no currently available technique is able to correlate femtosecond time scale transient microscopic data with local molecular structure and composition, thereby considerably limiting our ability to rationalize the origins of spatial variations in ultrafast electronic dynamics. Given the growing importance of these nanostructured materials and numerous indications of the existence and potential importance of spatial inhomogeneity, ${ }^{7,15-18}$ there is a clear need for moving ultrafast electronic and vibrational spectroscopy beyond the ensemble average and toward high spatial resolution.

As a result, considerable efforts have been made at combining femtosecond temporal with sub- $\mu \mathrm{m}$ spatial resolution. An ideal tool to address these aspects is (far-field) transient absorption microscopy (TAM), where a sample is photoexcited by a pump pulse with a duration typically of a few hundred femtoseconds followed, at a variable time delay, by a probe pulse that records the pump-induced signal response. Using a microscope objective-based imaging setup directly after the sample, this response can be directly mapped onto a camera, revealing lightinduced dynamics with sub- $\mu \mathrm{m}$ spatial resolution. ${ }^{17-19}$ Several experimental studies have demonstrated the enormous potential of TAM for a wide range of systems encompassing among others, solar cell materials, ${ }^{15,16,20-23}$ graphene sheets, $^{24-27}$ and carbon nanotubes ${ }^{28-30}$ as well as nanoplasmonic structures ${ }^{31-34}$ and the monitoring of drug delivery. ${ }^{35}$ Apart from a few exceptions, ${ }^{36}$ all experiments to date employ a traditional transmission microscope setup, in which both pulses pass through an illumination objective prior to interaction with the sample, limiting the achievable pulse durations to $\sim 100$ fs due to temporal dispersions introduced by the objective. ${ }^{17,18}$ Application of sophisticated pulse-shaping techniques have recently shown that it is possible to combine ultrashort near-IR pulses $(\sim 800 \mathrm{~nm}$, sub-10 fs), which have become ubiquitous in ultrafast spectroscopy, with high spatial resolution provided by high numerical aperture (NA) objectives. ${ }^{37}$ The extension toward the visible spectral region, where many of the most studied materials are strongly active, however, has been lacking.

To alleviate these shortcomings, we build on our recent work ${ }^{38,39}$ and present a wide-field femtosecond transient absorption microscope (fs-TAM) capable of delivering sub-10 fs pump and probe pulses to the sample while maintaining

Received: October 14, 2016

Accepted: November 10, 2016

Published: November 10, 2016 
(a)

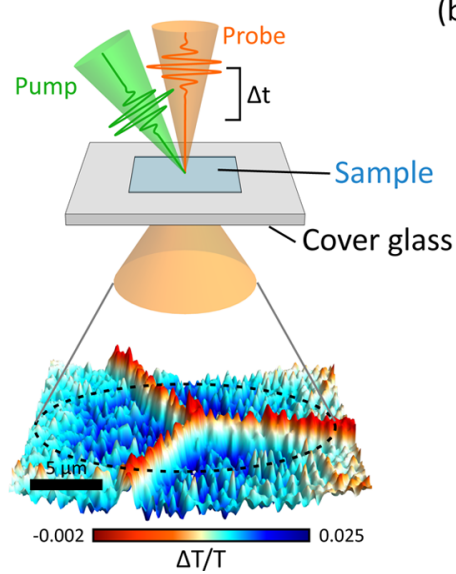

(b)

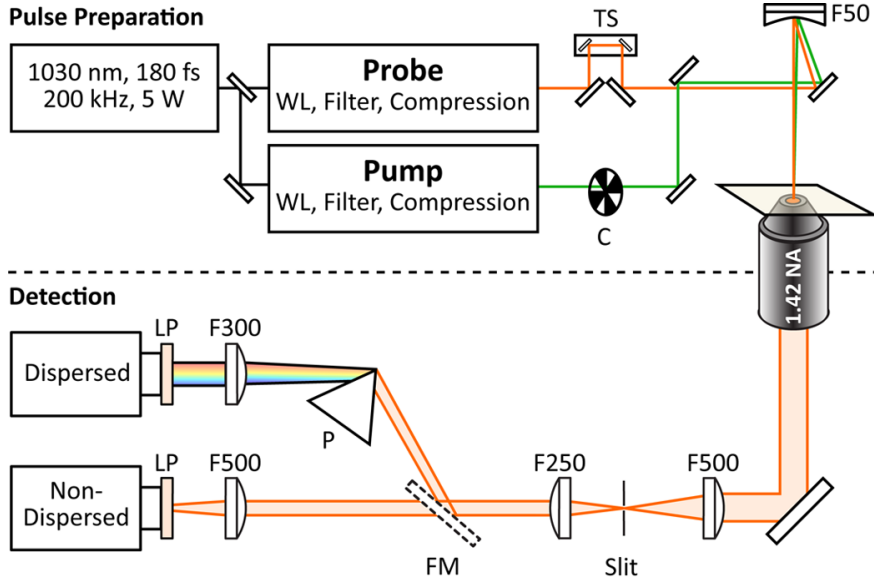

Figure 1. Concept and schematic experimental setup of wide-field fs-TAM. (a) A short focal length concave mirror focuses sub-10 fs pump and probe pulses onto a sample deposited on a cover glass slide. A high-NA objective collects both pulses to form a differential absorption image. The transient response is recorded with high spatial resolution to reveal the transient morphology of the sample, as illustrated here for a triple grain boundary in a $\mathrm{MAPbI}_{3-x} \mathrm{Cl}_{x}$ perovskite film. (b) Schematic of the setup based on white light (WL)-derived pump (500-640 nm) and probe (650$950 \mathrm{~nm}$ ) pulses. TS, translation stage; C, chopper; FM, flip mirror; P, prism; LP, $650 \mathrm{~nm}$ long-pass filter.

(a)

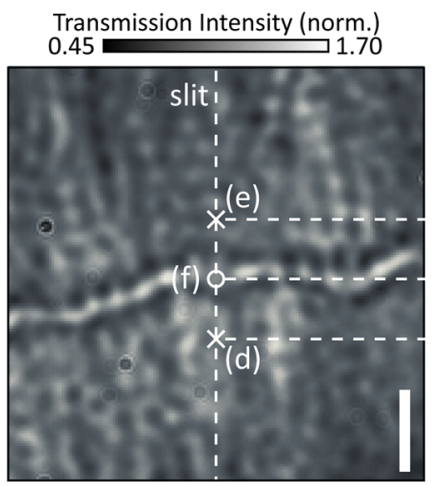

(b)

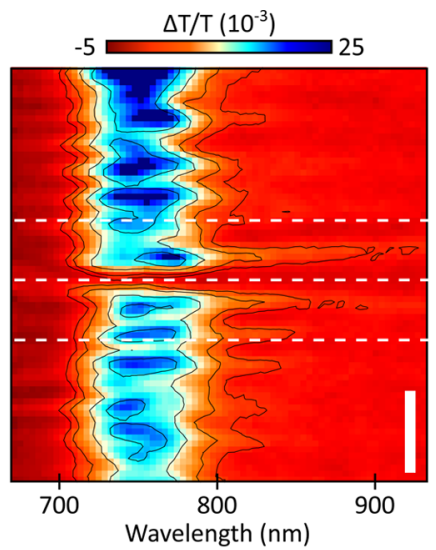

(d)

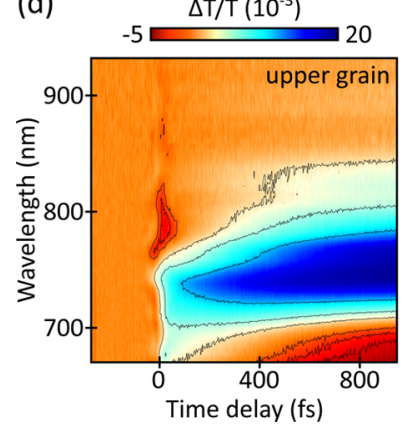

(e)

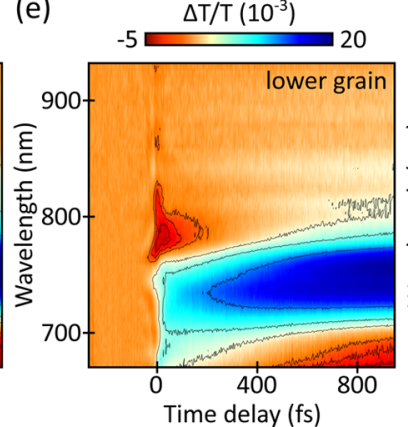

(f)

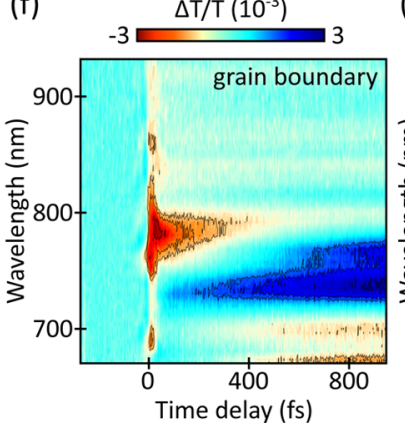

(c)

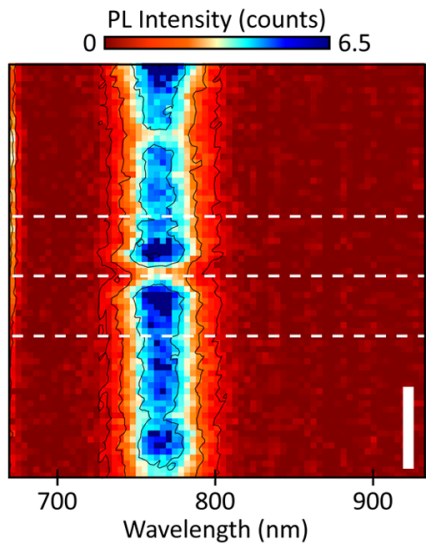

(g)

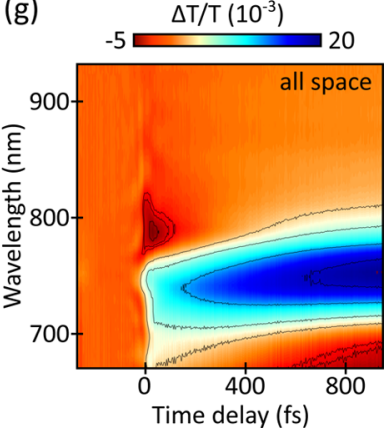

Figure 2. fs-TAM on $\mathrm{MAPbI}_{3-x} \mathrm{Cl}_{\mathrm{x}}$ perovskite films. (a) Normalized transmission image showing a horizontally aligned grain boundary. (b) Dispersed fs-TAM image at 1 ps pump-probe delay and (c) steady-state PL image of a diffraction-limited slice selected by the slit, as indicated in (a). (d-f) Transient absorption maps retrieved from the lower grain, the grain boundary, and the upper grain, as indicated in (a). (g) Transient absorption map averaged over a $20 \times 20 \mu \mathrm{m}^{2}$ region equivalent to an ensemble measurement. The scale bar in $(\mathrm{a}-\mathrm{c})$ is $2 \mu \mathrm{m}$. The original data were binned to $146.5 \mathrm{~nm} / \mathrm{px}$ in space and $3.8 \mathrm{~nm} / \mathrm{px}$ in wavelength. All experiments were carried out with the full probe bandwidth $(650-950 \mathrm{~nm})$ and fluences of 16 and $120 \mu \mathrm{J} / \mathrm{cm}^{2}$ for pump and probe pulses, respectively.

diffraction-limited spatial resolution (Figure 1a). The corresponding experimental setup is highly reminiscent of a standard transient absorption spectrometer where pump and probe pulses are focused onto a sample by a dispersion-free concave mirror (Figure $1 \mathrm{~b}){ }^{40,41}$ Rather than collecting the probe light using point- or spectrally-dispersed detection, we now collect the illumination beams with a high-NA microscope objective and subsequently image them onto a digital camera.
Alternatively, inserting a slit in an image plane combined with dispersion by a prism for maximum detection efficiency enables simultaneous $1 \mathrm{D}$ spatial and spectral imaging. ${ }^{38}$ Placement of the imaging optics after the pulses have interacted with the sample ensures that the strong dispersion induced by the objective does not affect the pulse duration at the sample. ${ }^{42}$ Consequently, the microscope simply images the transmission of the sample with a spatial resolution defined by $\lambda / 2 \mathrm{NA}$, where 
(a)

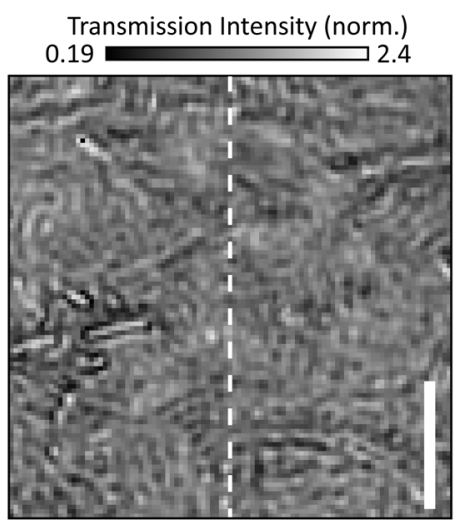

(d)

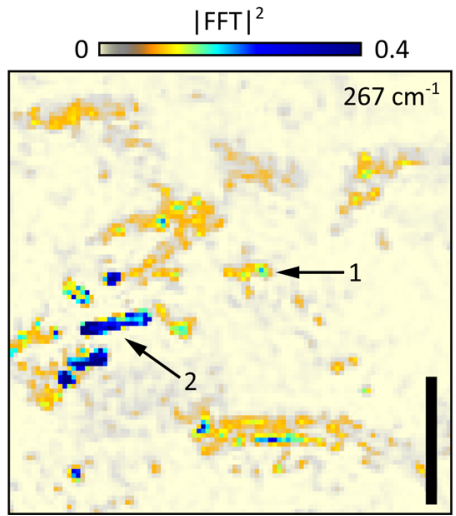

(b)

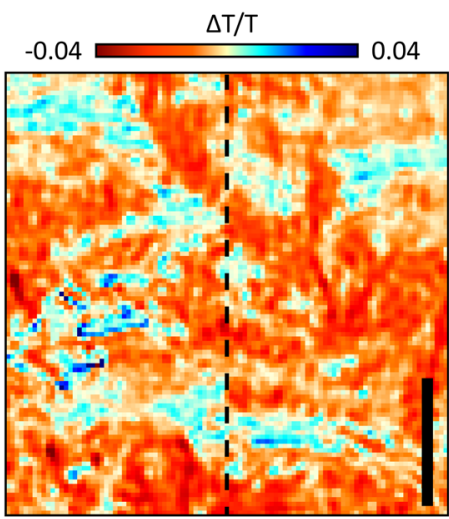

(e)

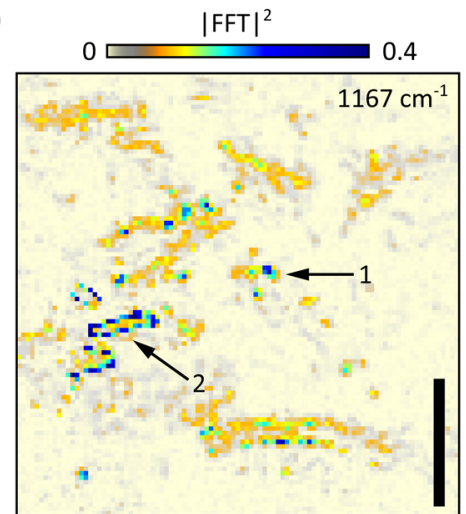

(c)

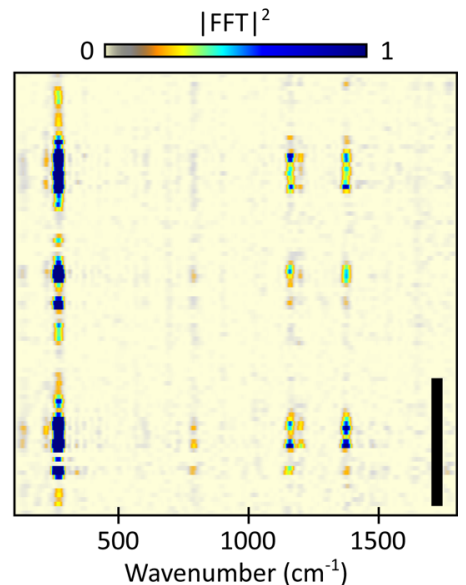

(f)

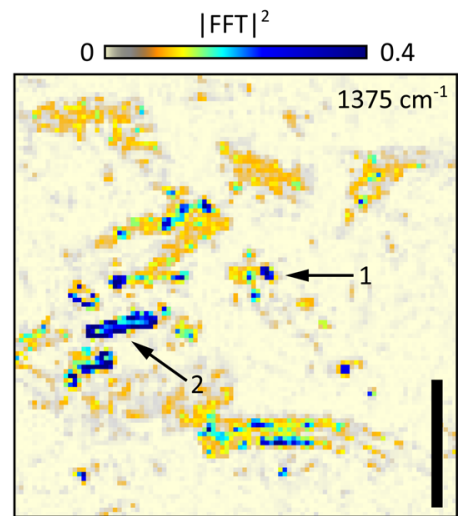

Figure 3. fs-TAM on aged TIPS pentacene films. (a) Normalized transmission image of a $15 \times 15 \mu \mathrm{m}^{2}$ area of an aged TIPS pentacene film. (b) Corresponding transient absorption image recorded at a pump-probe time delay of 1.25 ps. (c) Extracted Fourier transform power map for a diffraction-limited slice of the image as indicated by the vertically dashed line shown in (a) and (b). (d-f) Normalized Fourier transform power map of TIPS pentacene ground-state Raman bands at 267, 1167, and $1375 \mathrm{~cm}^{-1}$, respectively. Regions 1 and 2 illustrate large spatial differences in Fourier intensity. All fs-TAM images and corresponding spectra were recorded with a $740 \mathrm{~nm}$ band-pass filter $(\mathrm{fwhm}=10 \mathrm{~nm})$ in detection. The scale bar corresponds to $5 \mu \mathrm{m}$, and the data were binned to $175.9 \mathrm{~nm} / \mathrm{px}$. Pump and probe fluences were adjusted to 240 and $120 \mu \mathrm{J} / \mathrm{cm}^{2}$, respectively.

$\lambda$ is the carrier wavelength of the probe pulse and NA the numerical aperture of the objective. Modulation of the pump pulse by a chopper synchronized with the camera exposure thus returns differential transmission images of the sample. Delaying the probe relative to the pump pulse with a motorized translation stage retrieves transient kinetics completely analogous to that of standard pump-probe spectroscopy. ${ }^{40,41}$

To highlight the capabilities of fs-TAM, we begin by discussing the dispersed detection modality, applied to investigate the transient response of the mixed iodide-chloride methylammonium lead perovskite, $\mathrm{MAPbI}_{3-x} \mathrm{Cl}_{x}{ }^{5,43}$ The normalized transmission image of a grain boundary region (Figure 2a) recorded using a probe pulse covering 650-950 $\mathrm{nm}$ exhibits almost no absorption at the boundary but a strong reduction in the signal directly adjacent to it, most likely arising from strong scattering (Figure S1). Our perovskite grains appear to be regularly structured with brighter and darker absorption regions distributed throughout the grain (Figure 2a). We emphasize that this structure may originate from much smaller subdiffraction inhomogeneity that is not resolved by our optical microscope.

For dispersed detection, we close the slit positioned in the intermediate image plane to select a diffraction-limited slice of the image and direct the probe onto a prism for spectral dispersion along an axis perpendicular to the slit (Figure $1 \mathrm{~b}$ ). The corresponding transient absorption spectrum of our perovskite sample after photoexcitation with a $10 \mathrm{fs}$ pump pulse at $560 \mathrm{~nm}$ at a time delay of 1 ps exhibits a prominent bleach signal at $760 \mathrm{~nm}$, in agreement with previous reports (Figure 2b). ${ }^{44,45}$ Interestingly, the TA spectra exhibit large spectral variations as a function of space, indicating a significant underlying structural inhomogeneity even within the grain. To deduce whether this behavior is intrinsic to our sample, we blocked the probe pulse and measured the steady-state PL intensity generated by the pump pulse (Figure 2c). While some spectral changes remain evident, the spectrally resolved TAM image is significantly more inhomogeneous, suggesting a highly nonlocal environment of charge carriers directly after photoexcitation compared to the relaxed excited state measured with PL.

We now shift our focus toward the grain boundary, which shows almost no intensity in the fs-TAM or steady-state PL map (Figure $2 b, c$ ), in agreement with previous findings. ${ }^{46}$ To understand the transient kinetics, we compare spectrallyresolved TAM kinetics derived from the upper grain, the lower grain, and the grain boundary (Figure $2 \mathrm{~d}-\mathrm{f}$ ). All maps exhibit an early dispersive line shape with a photoinduced absorption at $780 \mathrm{~nm}$ and a bleach at $740 \mathrm{~nm}$, which evolves into a single bleach signal at $760 \mathrm{~nm}$ on the picosecond time scale. This behavior was previously assigned to band-gap renormalization occurring after above-band-gap excitation. ${ }^{44}$ Interestingly, the transient absorption response of the grain 
boundary (Figure 2f) shows a significantly reduced bleach contribution albeit with a similar intensity in the photoinduced absorption feature compared to the grain (Figure $2 \mathrm{~d}, \mathrm{e}$ ).

In addition, we can directly compare our fs-TAM results to ensemble TA spectroscopy experiments by averaging the transient absorption response over the full beam diameter (Figure $2 \mathrm{~g}$ ). The resulting TA map matches previous studies but lacks the characteristic signature of the grain boundary, emphasizing the importance of an imaging approach for samples with microscopic or nanoscopic inhomogeneity. Our results furthermore illustrate that the photoinduced absorption feature, corresponding to band-gap renormalization, grows in within $25 \mathrm{fs}$, irrespective of the spatial position within the sample, refining previous reports of a sub-100 fs time constant. $^{44}$ These initial results illustrate that the high spatiotemporal resolution promises to be an important asset in determining the optical responses of perovskite films to help uncover the reasons for their exceptional optoelectronic behavior. We remark that the unusual combination of a higher probe compared to pump power is a consequence of the interplay between maintaining a sufficiently low differential absorbance caused by the pump while maintaining sufficient photon counts in the probe to reveal the resulting signatures limited by shot noise. Although this is unconventional, we emphasize that the resulting transient absorbance spectra and kinetics are indistinguishable from those obtained at the ensemble level with a weaker probe compared to the pump, suggesting that our approach does not alter the observed dynamics.

To illustrate the chemical and structural sensitivity of our approach, we utilize the nondispersed imaging modality of fsTAM and apply it to investigate the transient morphology of TIPS pentacene films, ${ }^{47-49}$ which we have previously studied using vibronic spectroscopy with sub-10 fs resolution at the ensemble level. ${ }^{50}$ Here, we investigate films aged for 1 month under ambient light, which introduces considerable microscopic inhomogeneity to a previously smooth and homogeneous film (Figure S2), accompanied by local shifts of the absorption spectrum (Figure 3a). ${ }^{51}$ In addition, we introduced a $740 \mathrm{~nm}$ band-pass filter in the detection channel, to maximize our sensitivity to such local spectral shifts (see SI) because the macroscopic transient absorption spectrum exhibits a signinversion from a ground-state bleach signal to a photoinduced absorption signal at this wavelength with a steep gradient. ${ }^{50}$

The normalized transmission image of the aged film at 740 $\mathrm{nm}$ is spatially highly inhomogeneous (Figure 3a). The corresponding transient absorption image at a time delay of 1.25 ps following excitation by a $10 \mathrm{fs}$ pump pulse centered at $560 \mathrm{~nm}$ generally reproduces this inhomogeneity, which is dominated by photoinduced absorption signals (red, Figure $3 \mathrm{~b}$ ) with occasional ground-state bleach regions (blue, Figure $3 b$ ). A possible origin for this behavior is degradation of the TIPS pentacene film upon aging (Figures $\mathrm{S} 2-\mathrm{S} 4$ ), resulting in spatial regions where only tightly coupled pentacene molecules still exhibit dynamics close to the original ultrafast ground-state bleach response. This causes a significantly altered transient response more similar to uncoupled TIPS pentacene molecules, which is expected to blue-shift the transient response by $\sim 100$ nm (Figure S5). ${ }^{51}$

An intrinsic advantage of our ability to use extremely short pump and probe pulses in fs-TAM is the impulsive action of the pump pulse on the sample, which triggers synchronized molecular motion modulating the recorded time-domain signal via impulsive stimulated Raman scattering. ${ }^{52-54}$ Applying a Fourier transformation for recorded transients subsequently enables us to retrieve spatially-resolved Raman spectra. ${ }^{40,41}$ To illustrate this aspect of fs-TAM, we have selected a cut along the center of the image (dashed line in Figure 3a,b) and carried out a Fourier transform analysis for each recorded transient signal (Figure 3c). For homogenous samples, we would expect a homogeneous intensity distribution along the system's Raman frequencies (Figure S4). For the aged TIPS pentacene sample, however, we find large variations in Fourier intensity as a function of space. Comparing the relevant regions with the TAM image at 1.25 ps (Figure $3 \mathrm{~b}$ ) shows that ground-state bleaching signals result in measurable Fourier intensity, while photoinduced absorption signals lead to considerably reduced intensity. A comparison of the obtained Fourier spectra with a previously published time-domain ground-state spectrum further strengthens this correlation (see the SI for a more detailed analysis).

Beyond changes in the overall Fourier intensity, close inspection of the Fourier map reveals significant differences in the relative intensities of the individual bands (Figure 3c). To further emphasize this observation, we computed the Fourier transform power spectrum at each imaged pixel and mapped the spatial dependence of the three dominant groundstate bands located at 267, 1163, and $1375 \mathrm{~cm}^{-1}$ (Figure 3d-f). Despite overall similarity in the spatial distribution of the recorded Fourier intensity, we detect regions in which the relative Fourier intensities vary substantially as a function of space and wavenumber (compare regions 1 and 2 in Figure $3 \mathrm{~d}-\mathrm{f}$ ), highlighting the potential of fs-TAM to detect spatial inhomogeneity simultaneously with electronic and structural sensitivity (compare Figure $3 \mathrm{~b}$ and $\mathrm{d}-\mathrm{f}$ and see the $\mathrm{SI}$ ). We remark that the detection of vibrational coherences in the time domain is closely linked to the noise-floor of the experiment. Typical vibrational coherence amplitudes for the employed pulse parameters range from $\Delta T / T=10^{-3}$ to $10^{-4,40,41}$ while the current setup is routinely capable of achieving sensitivities below $\Delta T / T=10^{-4}$, making it widely applicable to a variety of systems (see the SI).

We have presented a fs-TAM capable of acquiring transient vibronic responses of nano-structured materials simultaneous sub-10 fs temporal and diffraction-limited spatial resolution. Initial experimental results for $\mathrm{MAPbI}_{3-x} \mathrm{Cl}_{x}$ perovskite and TIPS pentacene films demonstrate the potential of fs-TAM to investigate ultrafast dynamics following photoexcitation on microscopic and potentially nanoscopic length scales. The intrinsic ability of fs-TAM to record impulsively generated Raman spectra will prove extremely useful in determining not only the electronic but also the structural origins and outcomes of optical responses of materials. With the current experimental parameters, fs-TAM can achieve a shot-noise-limited localization precision of sub-30 nm, ${ }^{55,56}$ which, combined with the structural information, makes it ideally suited to study energy flow and transfer through nanoscale systems and across boundaries. We note that the current setup can be readily extended to include a super-resolution modality upon addition of a third saturation pulse. ${ }^{25}$ Furthermore, combination of focused pump with wide-field probe pulses will enable studies of spatially evolving vibronic dynamics with unprecedented simultaneous spatial and spectral resolution. 


\section{ASSOCIATED CONTENT}

\section{S Supporting Information}

The Supporting Information is available free of charge on the ACS Publications website at DOI: 10.1021/acs.jpclett.6b02387.

Experimental methods, data acquisition and analysis, sample preparation, and a detailed discussion on TIPS pentacene films as well as iSCAT images of perovskite blends (PDF)

\section{AUTHOR INFORMATION}

\section{Corresponding Author}

*E-mail: philipp.kukura@chem.ox.ac.uk.

ORCID

Christoph Schnedermann: 0000-0002-2841-8586

\section{Present Address}

${ }^{\S}$ A.S.D.: ICFO - Institut de Ciencies Fotoniques, The Barcelona Institute of Science \& Technology, 08860 Castelldefels (Barcelona), Spain.

\section{Notes}

The authors declare no competing financial interest.

\section{ACKNOWLEDGMENTS}

P.K. acknowledges funding by the EPSRC (EP/K006630/1) and a European Research Council (ERC) starting grant (NanoScope). A.R thanks the EPSRC and the Winton Program for the Physics of Sustainability for funding. C.S. gratefully acknowledges Adam Fineberg for his help in recording iSCAT images of perovskite films displayed in the Supporting Information.

\section{REFERENCES}

(1) Lu, L.; Zheng, T.; Wu, Q.; Schneider, A. M.; Zhao, D.; Yu, L. Recent Advances in Bulk Heterojunction Polymer Solar Cells. Chem. Rev. 2015, 115, 12666-12731.

(2) Rao, A.; Chow, P. C. Y.; Gélinas, S.; Schlenker, C. W.; Li, C.-Z.; Yip, H.-L.; Jen, A. K.-Y.; Ginger, D. S.; Friend, R. H. The Role of Spin in the Kinetic Control of Recombination in Organic Photovoltaics. Nature 2013, 500, 435-439.

(3) Gélinas, S.; Rao, A.; Kumar, A.; Smith, S. L.; Chin, A. W.; Clark, J.; van der Poll, T. S.; Bazan, G. C.; Friend, R. H. Ultrafast Long-Range Charge Separation in Organic Semiconductor Photovoltaic Diodes. Science 2014, 343, 512-516.

(4) Brenner, T. M.; Egger, D. A.; Kronik, L.; Hodes, G.; Cahen, D. Hybrid Organic-inorganic Perovskites: Low-Cost Semiconductors with Intriguing Charge-Transport Properties. Nat. Rev. Mater. 2016, 1, 15007.

(5) Stranks, S. D.; Snaith, H. J. Metal-Halide Perovskites for Photovoltaic and Light-Emitting Devices. Nat. Nanotechnol. 2015, 10, 391-402.

(6) Srimath Kandada, A. R.; Petrozza, A. Photophysics of Hybrid Lead Halide Perovskites: The Role of Microstructure. Acc. Chem. Res. 2016, 49, 536-544.

(7) Pazos-Outon, L. M.; Szumilo, M.; Lamboll, R.; Richter, J. M.; Crespo-Quesada, M.; Abdi-Jalebi, M.; Beeson, H. J.; Vru ini, M.; Alsari, M.; Snaith, H. J.; et al. Photon Recycling in Lead Iodide Perovskite Solar Cells. Science 2016, 351, 1430-1433.

(8) McNeill, C. R.; Ade, H. Soft X-Ray Characterisation of Organic Semiconductor Films. J. Mater. Chem. C 2013, 1, 187-201.

(9) Xing, G.; Mathews, N.; Sun, S.; Lim, S. S.; Lam, Y. M.; Gratzel, M.; Mhaisalkar, S.; Sum, T. C. Long-Range Balanced Electron- and Hole-Transport Lengths in Organic-Inorganic $\mathrm{CH}_{3} \mathrm{NH}_{3} \mathrm{PbI}_{3}$. Science 2013, 342, 344-347.

(10) Diao, Y.; Tee, B. C.-K.; Giri, G.; Xu, J.; Kim, D. H.; Becerril, H. A.; Stoltenberg, R. M.; Lee, T. H.; Xue, G.; Mannsfeld, S. C. B.; et al.
Solution Coating of Large-Area Organic Semiconductor Thin Films with Aligned Single-Crystalline Domains. Nat. Mater. 2013, 12, 665671.

(11) Chapman, H. N. X-Ray Imaging beyond the Limits. Nat. Mater. 2009, 8, 299-301.

(12) Ade, H.; Stoll, H. Near-Edge X-Ray Absorption Fine-Structure Microscopy of Organic and Magnetic Materials. Nat. Mater. 2009, 8, 281-290.

(13) Muller, D. A. Structure and Bonding at the Atomic Scale by Scanning Transmission Electron Microscopy. Nat. Mater. 2009, 8, 263-270.

(14) Yakunin, S.; Sytnyk, M.; Kriegner, D.; Shrestha, S.; Richter, M.; Matt, G. J.; Azimi, H.; Brabec, C. J.; Stangl, J.; Kovalenko, M. V.; et al. Detection of X-Ray Photons by Solution-Processed Lead Halide Perovskites. Nat. Photonics 2015, 9, 444-449.

(15) Polli, D.; Grancini, G.; Clark, J.; Celebrano, M.; Virgili, T.; Cerullo, G.; Lanzani, G. Nanoscale Imaging of the Interface Dynamics in Polymer Blends by Femtosecond Pump-Probe Confocal Microscopy. Adv. Mater. 2010, 22, 3048-3051.

(16) Wan, Y.; Guo, Z.; Zhu, T.; Yan, S.; Johnson, J.; Huang, L. Cooperative Singlet and Triplet Exciton Transport in Tetracene Crystals Visualized by Ultrafast Microscopy. Nat. Chem. 2015, 7, 785792.

(17) Davydova, D.; de la Cadena, A.; Akimov, D.; Dietzek, B. Transient Absorption Microscopy: Advances in Chemical Imaging of Photoinduced Dynamics. Laser Photon. Rev. 2016, 10, 62-81.

(18) Fischer, M. C.; Wilson, J. W.; Robles, F. E.; Warren, W. S. Invited Review Article: Pump-Probe Microscopy. Rev. Sci. Instrum. 2016, 87, 031101.

(19) Grumstrup, E. M.; Gabriel, M. M.; Cating, E. E. M.; Van Goethem, E. M.; Papanikolas, J. M. Pump-probe Microscopy: Visualization and Spectroscopy of Ultrafast Dynamics at the Nanoscale. Chem. Phys. 2015, 458, 30-40.

(20) Virgili, T.; Grancini, G.; Molotokaite, E.; Suarez-Lopez, I.; Rajendran, S. K.; Liscio, A.; Palermo, V.; Lanzani, G.; Polli, D.; Cerullo, G. Confocal Ultrafast Pump-probe Spectroscopy: A New Technique to Explore Nanoscale Composites. Nanoscale 2012, 4, 2219-2226.

(21) Grancini, G.; Polli, D.; Fazzi, D.; Cabanillas-Gonzalez, J.; Cerullo, G.; Lanzani, G. Transient Absorption Imaging of P3HT:PCBM Photovoltaic Blend: Evidence For Interfacial Charge Transfer State. J. Phys. Chem. Lett. 2011, 2, 1099-1105.

(22) Wong, C. Y.; Cotts, B. L.; Wu, H.; Ginsberg, N. S. Exciton Dynamics Reveal Aggregates with Intermolecular Order at Hidden Interfaces in Solution-Cast Organic Semiconducting Films. Nat. Commun. 2015, 6, 5946.

(23) Guo, Z.; Manser, J. S.; Wan, Y.; Kamat, P. V.; Huang, L. Spatial and Temporal Imaging of Long-Range Charge Transport in Perovskite Thin Films by Ultrafast Microscopy. Nat. Commun. 2015, 6, 7471.

(24) Huang, L.; Hartland, G. V.; Chu, L.-Q.; Luxmi; Feenstra, R. M.; Lian, C.; Tahy, K.; Xing, H. Ultrafast Transient Absorption Microscopy Studies of Carrier Dynamics in Epitaxial Graphene. Nano Lett. 2010, 10, 1308-1313.

(25) Wang, P.; Slipchenko, M. N.; Mitchell, J.; Yang, C.; Potma, E. O.; Xu, X.; Cheng, J.-X. Far-Field Imaging of Non-Fluorescent Species with Subdiffraction Resolution. Nat. Photonics 2013, 7, 449-453.

(26) Gao, B.; Hartland, G.; Fang, T.; Kelly, M.; Jena, D.; Xing, H.; Huang, L. Studies of Intrinsic Hot Phonon Dynamics in Suspended Graphene by Transient Absorption Microscopy. Nano Lett. 2011, 11, 3184-3189.

(27) Graham, M. W.; Shi, S.-F.; Wang, Z.; Ralph, D. C.; Park, J.; McEuen, P. L. Transient Absorption and Photocurrent Microscopy Show That Hot Electron Supercollisions Describe the Rate-Limiting Relaxation Step in Graphene. Nano Lett. 2013, 13, 5497-5502.

(28) Jung, Y.; Slipchenko, M. N.; Liu, C. H.; Ribbe, A. E.; Zhong, Z.; Yang, C.; Cheng, J.-X. Fast Detection of the Metallic State of Individual Single-Walled Carbon Nanotubes Using a TransientAbsorption Optical Microscope. Phys. Rev. Lett. 2010, 105, 217401. 
(29) Chen, T.; Lu, F.; Streets, A. M.; Fei, P.; Quan, J.; Huang, Y. Optical Imaging of Non-Fluorescent Nanodiamonds in Live Cells Using Transient Absorption Microscopy. Nanoscale 2013, 5, 4701.

(30) Gao, B.; Hartland, G. V.; Huang, L. Transient Absorption Spectroscopy of Excitons in an Individual Suspended Metallic Carbon Nanotube. J. Phys. Chem. Lett. 2013, 4, 3050-3055.

(31) Huang, L.; Cheng, J.-X. Nonlinear Optical Microscopy of Single Nanostructures. Annu. Rev. Mater. Res. 2013, 43, 213-236.

(32) Yu, K.; Devadas, M. S.; Major, T. A.; Lo, S. S.; Hartland, G. V. Surface Plasmon Polariton Propagation and Coupling in Gold Nanostructures. J. Phys. Chem. C 2014, 118, 8603-8609.

(33) Gabriel, M. M.; Kirschbrown, J. R.; Christesen, J. D.; Pinion, C. W.; Zigler, D. F.; Grumstrup, E. M.; Mehl, B. P.; Cating, E. E. M.; Cahoon, J. F.; Papanikolas, J. M. Direct Imaging of Free Carrier and Trap Carrier Motion in Silicon Nanowires by Spatially-Separated Femtosecond Pump-Probe Microscopy. Nano Lett. 2013, 13, 13361340.

(34) Hartland, G. V. Ultrafast Studies of Single Semiconductor and Metal Nanostructures through Transient Absorption Microscopy. Chem. Sci. 2010, 1, 303.

(35) Min, W.; Lu, S.; Chong, S.; Roy, R.; Holtom, G. R.; Xie, X. S. Imaging Chromophores with Undetectable Fluorescence by Stimulated Emission Microscopy. Nature 2009, 461, 1105-1109.

(36) Seo, M.; Boubanga-Tombet, S.; Yoo, J.; Ku, Z.; Gin, A. V.; Picraux, S. T.; Brueck, S. R. J.; Taylor, A. J.; Prasankumar, R. P. Ultrafast Optical Wide Field Microscopy. Opt. Express 2013, 21, 8763.

(37) Pawłowska, M.; Goetz, S.; Dreher, C.; Wurdack, M.; Krauss, E.; Razinskas, G.; Geisler, P.; Hecht, B.; Brixner, T. Shaping and Spatiotemporal Characterization of Sub-10-fs Pulses Focused by a High-NA Objective. Opt. Express 2014, 22, 31496-31510.

(38) Weigel, A.; Sebesta, A.; Kukura, P. Dark Field Microspectroscopy with Single Molecule Fluorescence Sensitivity. ACS Photonics 2014, 1, 848-856.

(39) Weigel, A.; Sebesta, A.; Kukura, P. Shaped and FeedbackControlled Excitation of Single Molecules in the Weak-Field Limit. J. Phys. Chem. Lett. 2015, 6, 4032-4037.

(40) Liebel, M.; Schnedermann, C.; Wende, T.; Kukura, P. Principles and Applications of Broadband Impulsive Vibrational Spectroscopy. J. Phys. Chem. A 2015, 119, 9506-9517.

(41) Kuramochi, H.; Takeuchi, S.; Tahara, T. Femtosecond TimeResolved Impulsive Stimulated Raman Spectroscopy Using Sub-7-fs Pulses: Apparatus and Applications. Rev. Sci. Instrum. 2016, 87, 043107.

(42) Pollard, W. T.; Mathies, R. A. Analysis of Femtosecond Dynamic Absorption Spectra of Nonstationary States. Annu. Rev. Phys. Chem. 1992, 43, 497-523.

(43) Colella, S.; Mosconi, E.; Fedeli, P.; Listorti, A.; Gazza, F.; Orlandi, F.; Ferro, P.; Besagni, T.; Rizzo, A.; Calestani, G.; et al. $\mathrm{MAPbI}_{3-x} \mathrm{Cl}_{\mathrm{X}}$ Mixed Halide Perovskite for Hybrid Solar Cells: The Role of Chloride as Dopant on the Transport and Structural Properties. Chem. Mater. 2013, 25, 4613-4618.

(44) Price, M. B.; Butkus, J.; Jellicoe, T. C.; Sadhanala, A.; Briane, A.; Halpert, J. E.; Broch, K.; Hodgkiss, J. M.; Friend, R. H.; Deschler, F. Hot-Carrier Cooling and Photoinduced Refractive Index Changes in Organic-inorganic Lead Halide Perovskites. Nat. Commun. 2015, 6, 8420.

(45) Sharma, V.; Aharon, S.; Gdor, I.; Yang, C.; Etgar, L.; Ruhman, S. New Insights into Exciton Binding and Relaxation from High Time Resolution Ultrafast Spectroscopy of $\mathrm{CH}_{3} \mathrm{NH}_{3} \mathrm{PbI}_{3}$ and $\mathrm{CH}_{3} \mathrm{NH}_{3} \mathrm{PbBr}_{3}$ Films. J. Mater. Chem. A 2016, 4, 3546-3553.

(46) de Quilettes, D. W.; Vorpahl, S. M.; Stranks, S. D.; Nagaoka, H.; Eperon, G. E.; Ziffer, M. E.; Snaith, H. J.; Ginger, D. S. Impact of Microstructure on Local Carrier Lifetime in Perovskite Solar Cells. Science 2015, 348, 683-686.

(47) Wilson, M. W. B.; Rao, A.; Clark, J.; Kumar, R. S. S.; Brida, D.; Cerullo, G.; Friend, R. H. Ultrafast Dynamics of Exciton Fission in Polycrystalline Pentacene. J. Am. Chem. Soc. 2011, 133, 11830-11833.

(48) Yost, S. R.; Lee, J.; Wilson, M. W. B.; Wu, T.; McMahon, D. P.; Parkhurst, R. R.; Thompson, N. J.; Congreve, D. N.; Rao, A.; Johnson,
K.; et al. A Transferable Model for Singlet-Fission Kinetics. Nat. Chem. 2014, 6, 492-497.

(49) Bakulin, A. A.; Morgan, S. E.; Kehoe, T. B.; Wilson, M. W. B.; Chin, A. W.; Zigmantas, D.; Egorova, D.; Rao, A. Real-Time Observation of Multiexcitonic States in Ultrafast Singlet Fission Using Coherent 2D Electronic Spectroscopy. Nat. Chem. 2015, 8, 1623.

(50) Musser, A. J.; Liebel, M.; Schnedermann, C.; Wende, T.; Kehoe, T. B.; Rao, A.; Kukura, P. Evidence for Conical Intersection Dynamics Mediating Ultrafast Singlet Exciton Fission. Nat. Phys. 2015, 11, 352357.

(51) Walker, B. J.; Musser, A. J.; Beljonne, D.; Friend, R. H. Singlet Exciton Fission in Solution. Nat. Chem. 2013, 5, 1019-1024.

(52) Ruhman, S.; Joly, A. G.; Nelson, K. A. Coherent Molecular Vibrational Motion Observed in the Time Domain through Impulsive Stimulated Raman Scattering. IEEE J. Quantum Electron. 1988, 24, 460-469.

(53) Ruhman, S.; Kohler, B.; Joly, A. G.; Nelson, K. A. Molecular Dynamics in Liquids from Femtosecond Time-Resolved Impulsive Stimulated Scattering. IEEE J. Quantum Electron. 1988, 24, 470-481.

(54) Dorfman, K. E.; Fingerhut, B. P.; Mukamel, S. Time-Resolved Broadband Raman Spectroscopies: A Unified Six-Wave-Mixing Representation. J. Chem. Phys. 2013, 139, 124113.

(55) Ortega-Arroyo, J.; Kukura, P. Interferometric Scattering Microscopy (iSCAT): New Frontiers in Ultrafast and Ultrasensitive Optical Microscopy. Phys. Chem. Chem. Phys. 2012, 14, 15625-15636.

(56) Ortega Arroyo, J.; Cole, D.; Kukura, P. Interferometric Scattering Microscopy and Its Combination with Single-Molecule Fluorescence Imaging. Nat. Protoc. 2016, 11, 617-633. 